\title{
Brain tumors and driving
}

\author{
Kerrie Leanne McDonald
}

Received: 13 November 2010/ Accepted: 2 December 2010/Published online: 15 December 2010

(C) The Author(s) 2010. This article is published with open access at Springerlink.com

\section{To the Editor:}

Referring to the article: "Current practices of driving restriction implementation for patients with brain tumors" by Thomas et al., published in your journal in October 2010 (published online) [1], I am writing to inform readers of the recent initiative of the Cancer Institute NSW Oncology Working Group for Neuro-oncology (NSWOG, Australia) to address the serious issue of driving a motor vehicle after diagnosis of a brain tumor. Driving a motor vehicle is a complex task and patients diagnosed with a brain tumor are at a higher accident risk because of the significant neurocognitive deficits associated with the disease. As the authors pointed out, seizures, memory lapses, and cognitive dysfunction can result from both benign and malignant brain tumors, placing the driver, its passengers and innocent bystanders in danger. In the USA, it is up to individual States to enforce reporting of conditions which impair driving. In Australia, the driver licensing authority (DLA) holds the legal responsibility to determine eligibility to drive, and the treating clinician's role to provide relevant medical information [2]. In practice however, the responsibility lies with the clinician. Australia is fortunate to have formalized recommendations, but despite the release of "Assessing Fitness to Drive" by Austroads (guidelines approved by all Australian licensing authorities), patients are often given conflicting advice with regards to their ability or fitness to drive. This is consistent with the findings and conclusions generated by Thomas and

\section{K. L. McDonald ( $\square)$}

Prince of Wales Clinical School, Adult Cancer Program, Cure For Life Neuro-oncology Group, Lowy Cancer Research Centre, University of New South Wales, Sydney, NSW, Australia e-mail: k.mcdonald@unsw.edu.au colleagues. In an Australian survey of practicing neurosurgeons, neurologists and radiation oncologists $(n=194)$, $73 \%$ of respondents were not aware of any current guidelines [3]. Unanimously, the conclusion and recommendation reached by both the USA and Australian surveys were to form a multidisciplinary working party to develop more specific guidelines [1,3].

In response to these studies, a panel of experts in neurosurgery, medical oncology, occupational therapy, rehabilitation and allied nursing staff generated two information brochures- one for clinicians and another designed for patients. The Brain Tumors and Driving: A guide for Clinicians brochure highlights a simplified algorithm to help the clinician provide informed advice in regard to driving a motor vehicle to their patients. The algorithm begins with confirmed diagnosis of a brain tumor. The next step of the algorithm decision tree pertains to treatment and physiology: Has the patient had a craniotomy? If this is the case, it is an Australian recommendation to not drive for 3 months. After the 3 months, the algorithm addresses the presentation of significant deficits, borderline deficits or no deficits? In the presence of a significant deficit, no driving is permitted. In the case of a borderline deficit, referral to an occupation therapist is recommended and fitness to drive assessed. For patients with no deficit, the green light is given for driving. This algorithm does not discriminate between benign and malignant tumors since seizure activity is independent of grade. The algorithm is predominantly based on deficit presentation. Guidelines for seizures are also presented. Quite often, conflict can occur between the patient and clinician delivering the news that they are not fit to drive. The brochure for clinicians provides useful information in regard to how to talk to patients and their families and tips about licence reinstatement and help lines. In addition, the 
NSWOG working group developed a second brochure to give to patients. The Brain Tumors and Driving: A guide for patients and carers outlines in layman's terms the reasons why they should not be driving, what the responsibilities of the clinician are, what the process is for returning to driving and a list of alternatives in the case where the patient cannot drive. Driving is a privilege, not a right and driving by people with brain tumors is a serious issue. The two Brain Tumor and Driving Brochures can be found at http://www.cancerinstitute. org.au/cancer_inst/nswog/groups/neuro1.html.

Open Access This article is distributed under the terms of the Creative Commons Attribution Noncommercial License which permits any noncommercial use, distribution, and reproduction in any medium, provided the original author(s) and source are credited.

\section{References}

1. Thomas S, Mehta M, Kuo JS, Robins I, Khuntia D (2010) Current practices of driving restriction implementation for patients with brain tumors. J Neurooncol. doi:10.1007/s11060-010-0439-7

2. Somerville ER, Black AB, Dunne JW (2010) Driving to distraction: certification of fitness to drive with epilepsy. Med J Aust 192:342-344

3. Chin YS, Jayamohan J, Clouston P, Gebski V, Cakir B (2004) Driving and patients with brain tumours: a postal survey of neurosurgeons, neurologists and radiation oncologists. J Clin Neurosci 11:471-474 